\title{
Effect of strain rate on the compressive deformation behaviors of lotus-type porous copper
}

\author{
Xin-hua Liu ${ }^{1.2)}$, Hai-you Huang ${ }^{1.2)}$, and Jian-xin Xie ${ }^{1.2)}$ \\ 1) Institute for advanced Materials and Technology, University of Science and Technology Beijing, Beijing 100083, China \\ 2) Key Laboratory for Advanced Materials Processing (MOE), University of Science and Technology Beijing, Beijing 100083, China \\ (Received: 19 August 2013; revised: 11 March 2014; accepted: 13 March 2014)
}

\begin{abstract}
Lotus-type porous copper was fabricated by unidirectional solidification, and compressive experiments were subsequently conducted in the strain rate range of $10^{-3}-2400 \mathrm{~s}^{-1}$ with the compressive direction parallel to the pores. A GLEEBLE-1500 thermal-mechanical simulation system and a split Hopkinson pressure bar (SHPB) were used to investigate the effect of strain rate on the compressive deformation behaviors of lotus-type porous copper. The influence mechanism of strain rate was also analyzed by the strain-controlling method and by high-speed photography. The results indicated that the stress-strain curves of lotus-typed porous copper consist of a linear elastic stage, a plateau stage, and a densification stage at various strain rates. At low strain rate $\left(<1.0 \mathrm{~s}^{-1}\right)$, the strain rate had little influence on the stress-strain curves; but when the strain rate exceeded $1.0 \mathrm{~s}^{-1}$, it was observed to strongly affect the plateau stage, showing obvious strain-rate-hardening characteristics. Strain rate also influenced the densification initial strain. The densification initial strain at high strain rate was less than that at low strain rate. No visible inhomogeneous deformation caused by shockwaves was observed in lotus-type porous copper during high-strain-rate deformation. However, at high strain rate, the bending deformation characteristics of the pore walls obviously differed from those at low strain rate, which was the main mechanism by which the plateau stress exhibited strain-rate sensitivity when the strain rate exceeded a certain value and exhibited less densification initial strain at high strain rate.
\end{abstract}

Keywords: porous materials; copper; directional solidification; strain rate sensitivity; deformation modes; stress-strain curves

\section{Introduction}

Porous metals are novel engineering materials with lower density, larger deformation under constant compression stress, larger surface area, and good permeability compared with their dense counterpart metals. Porous metals have been used as light-weight structural components, heat-exchangers, filters, sound absorption components, bioengineering materials, etc. [1-3]. In particular, porous metals' low density and special response characteristics to compression stress make them suitable for use in the transportation, aerospace, and military equipment fields as excellent energy absorption materials and security/protection materials. In recent years, a new kind of porous metal called lotus-type porous metal has been rapidly developed [4]. Compared with foam metal, which has a spherical or subsphaeroidal porous structure, lotus-type porous metal has long cylindrical pores regularly arrayed along a special direction and ex- hibits excellent mechanical properties, such as no obvious stress concentration, a high tensile/compressive strength, and a high stress platform. Thus, lotus-type porous metal has attracted increasing interest from scientists and engineers [5-9].

The mechanical characteristics of porous materials at various strain rates are important reference information for the materials selection and structure design of energy-absorption and security/protection parts. In recent years, some researchers have studied these materials extensively [10-14]. The phenomenon of the mechanical characteristics of materials obviously increasing with increasing strain rate is called strain-rate sensitivity, which is one of the most important characteristics of porous materials. However, the literature contains some inconsistent results related to the strain-rate sensitivity of porous metals. Deshpande and Fleck [11] investigated the mechanical properties of two kinds of foam aluminum at strain rates from $10^{-3} \mathrm{~s}^{-1}$ to 5000 
$\mathrm{s}^{-1}$ and observed no obvious strain-rate sensitivity of plateau stress. Dannemann and Lankford [12] observed that, although the compression strength of closed-cell foam aluminum did not show obvious strain-rate sensitivity, its plateau stress exhibited strain-rate sensitivity at strain rates from 400 $\mathrm{s}^{-1}$ to $2500 \mathrm{~s}^{-1}$. Koichi and Yoshihiro [15] indicated that foam $\mathrm{Zn}-22 \mathrm{Al}$ alloy exhibited greater strain-rate sensitivity compared to traditional foam aluminum under compression stress and attributed this phenomenon to the fact that the $\mathrm{Zn}-22 \mathrm{Al}$ alloy used in their study contained fine equiaxed crystals and exhibited superplasticity. Feng et al. [16] investigated the effect of strain rate on the compression properties of foam aluminum alloys and observed that the yield stress increased with increasing strain rate at the high-strain-rate stage and that the strain-rate sensitivity increased as the relative density increased.

In previous studies, we have investigated the mechanical properties of lotus-type porous copper under quasi-static compressive deformation at a strain rate less than $10^{-1} \mathrm{~s}^{-1}$ and observed no obvious strain-rate sensitivity [17-18]. However, our recent research has indicated that strain-rate sensitivity could be observed when the strain rate exceeded a certain value. In the present paper, the compressive deformation behaviors of lotus-type porous copper were investigated over a wide strain-rate range of $10^{-3}$ to $2400 \mathrm{~s}^{-1}$ to explore the effect of the strain rate on the deformation behaviors and its mechanism.

\section{Experimental}

\subsection{Experimental materials}

The unidirectional solidification method was used to fab- ricate lotus-type porous copper; the apparatus is illustrated in Fig. 1. The fabrication process of lotus-type copper was as follows: First, the solidification device was evacuated and then back-filled with hydrogen. Second, the copper was melted in a graphite crucible at $1200^{\circ} \mathrm{C}$ and maintained at this temperature for $0.5 \mathrm{~h}$ to make gas saturated in molten copper. An alumina casting mold with an inner diameter of $50 \mathrm{~mm}$ and a height of $170 \mathrm{~mm}$ was used. After the temperature was increased to the set value, the graphite stopper was removed and the molten copper was cast into the alumina casting mold through a funnel. The lotus-type porous copper was subsequently formed by unidirectional solidification. The fabricated lotus-type porous copper bar is shown in Fig. 2.

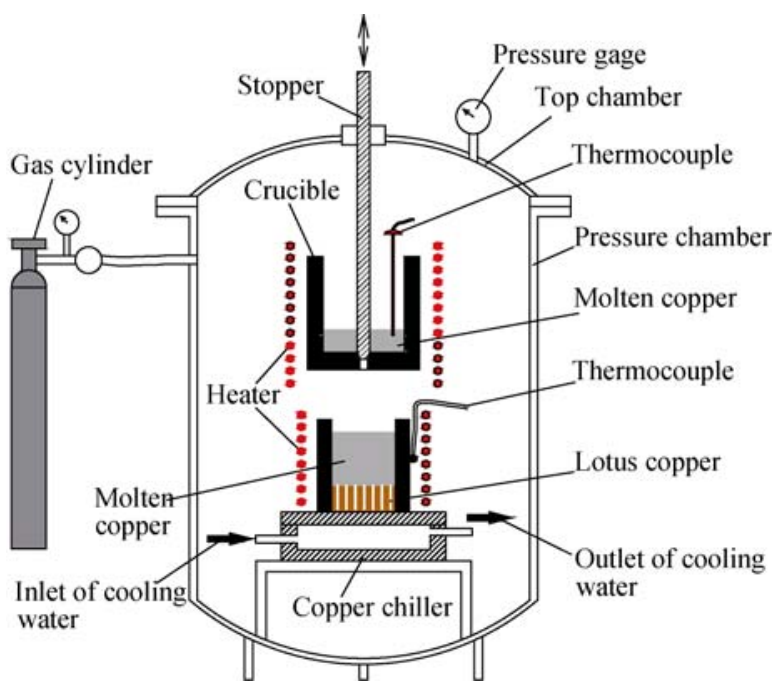

Fig. 1. Schematic diagram of high-pressured unidirectional solidification processing for fabricating lotus-type porous metals.

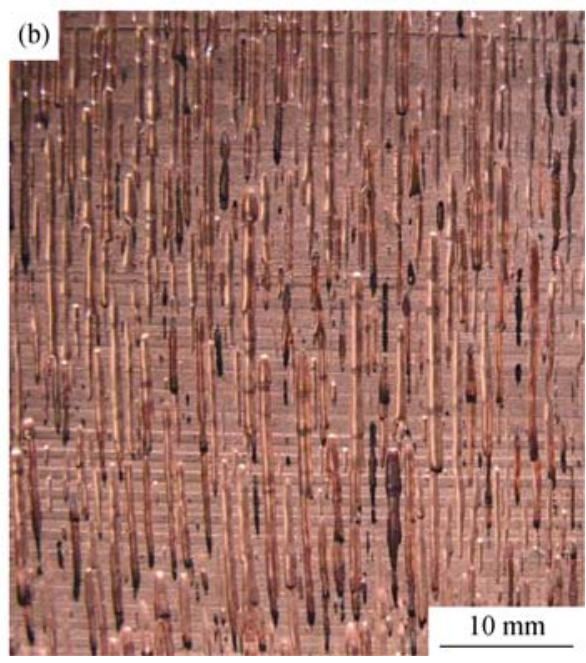

Fig. 2. Section morphologies of a lotus-type porous copper bar: (a) transverse; (b) longitudinal. 
Cubic specimens with dimensions of $10 \mathrm{~mm} \times 10 \mathrm{~mm} \times$ $10 \mathrm{~mm}$ were cut from the lotus-type porous copper bar by linear cutting to prepare specimens for the compression tests. The four lateral surfaces of the specimen were parallel to the axis or longitudinal direction (LD) of the cylindrical of pores, and the upper and lower surfaces were vertical to the LD. The weight and length of the cubic specimens were precisely measured on a fine balance and with a Vernier caliper, respectively, after they were cleaned by ultrasonication in ethanol solution. The relative density $\rho_{\mathrm{r}}$ and porosity $P$ were calculated according to Eqs. (1) and (2), respectively:

$$
\begin{aligned}
& \rho_{\mathrm{r}}=\frac{M / V}{\rho_{\mathrm{d}}}=\frac{M}{V \rho_{\mathrm{d}}} \\
& P=\left(1-\rho_{\mathrm{r}}\right) \times 100 \%
\end{aligned}
$$

where $M$ is the weight of the lotus-type porous copper
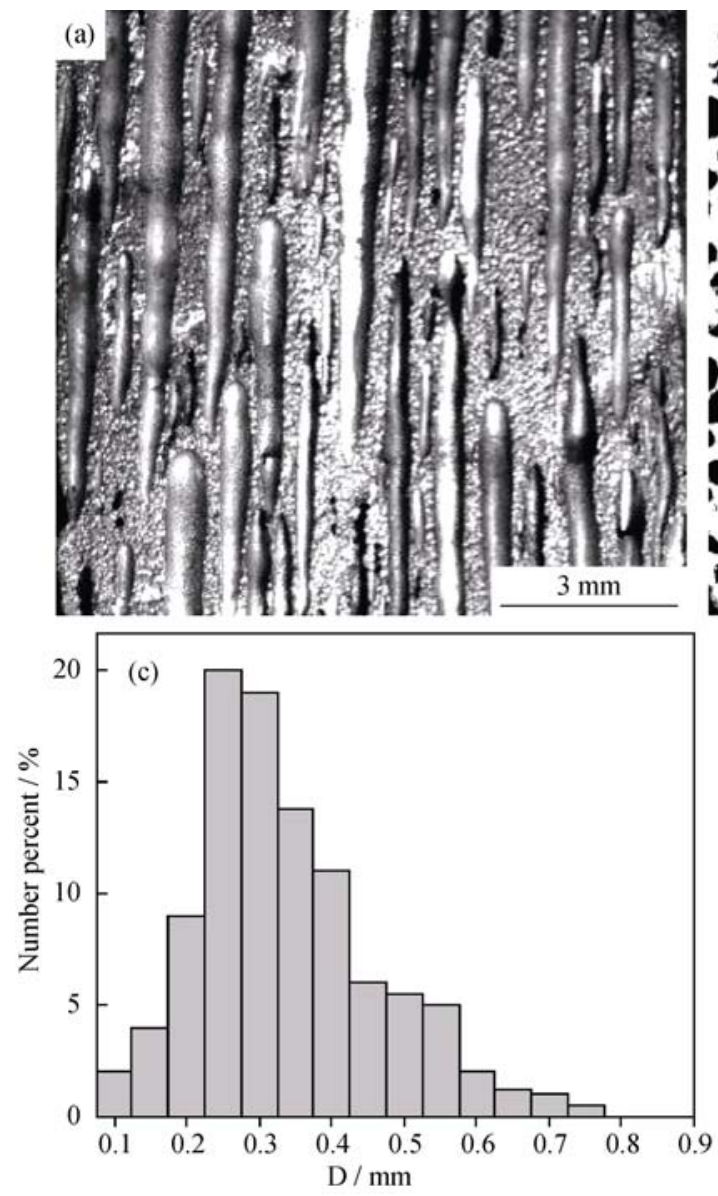

specimen $(\mathrm{g}), V$ is the volume of the specimen $\left(\mathrm{cm}^{3}\right)$, and $\rho_{\mathrm{d}}$ is the density of pure copper $\left(8.9 \mathrm{~g} / \mathrm{cm}^{3}\right)$.

The Image J software was used to statistically analyze the pore structure of the compression-test specimens. The morphologies of the cross-section and the longitudinal section of the as-cast lotus-type porous copper specimens are shown in Figs. 3(a) and 3(b), respectively; the images indicate that the distribution of the pores is homogeneous in both the cross-sections and the longitudinal sections. The pore shape is almost cylindrical, and the growth direction is approximately straight. Fig. 3(c) is the pore diameter distribution diagram, as analyzed by the Image $\mathbf{J}$ software; it indicates that the values of pore diameter lie in the range of $0.1-0.75$ $\mathrm{mm}$ and that $80 \%$ of them distribute between $0.15 \mathrm{~mm}$ and $0.55 \mathrm{~mm}$. The relative density is 0.45 , and the porosity is about $55 \%$.

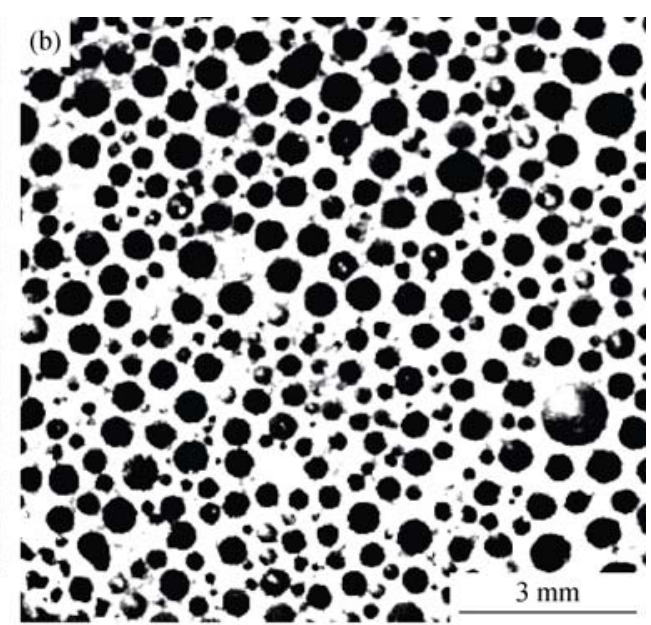

Fig. 3. Morphologies of (a) the longitudinal section and (b) the cross section of the as-cast lotus-type porous copper specimen and (c) its pore diameter distribution diagram.

\subsection{Experimental methods}

The compression experiments were conducted at room temperature. All compression directions were along the LD, as shown in Fig. 4. A GLEEBLE-1500 thermal-mechanical simulation system and a direct impact split Hopkinson pre- ssure bar (SHPB) were used for the low-strain-rate $\left(10^{-3}-30\right.$ $\mathrm{s}^{-1}$ ) compression tests (LSRCTs) and high-strain- rate (800-2400 s ${ }^{-1}$ ) compression tests (HSRCTs), respectively.

For the LSRCTs, the specimens were compressed at a strain rate of $10^{-3} \mathrm{~s}^{-1}, 10^{-1} \mathrm{~s}^{-1}, 1.0 \mathrm{~s}^{-1}$, and $30 \mathrm{~s}^{-1}$ to a deformation strain of 0.8 . Load-displacement curves were di- 
rectly obtained by the thermomechanical simulation system. The engineering stress-engineering strain curves were subsequently calculated from the load-displacement curves.
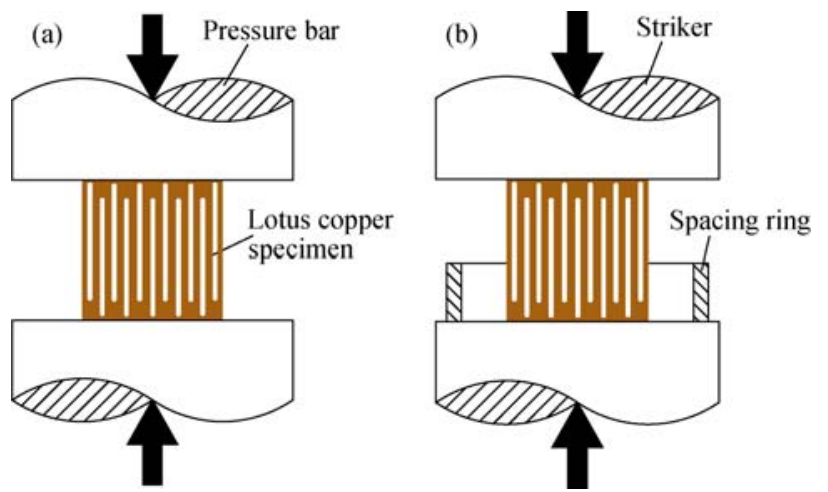

Fig. 4. Schematic diagrams of compression experiments: (a) LSRCT; (b) HSRCT.

Fig. 5 illustrates a schematic diagram of the direct-impact SHPB, which is suitable for large deformation strain compression tests $[13,19]$. In the HSRCTs, strain rates of $857 \mathrm{~s}^{-1}$, $1583 \mathrm{~s}^{-1}$, and $2384 \mathrm{~s}^{-1}$ and a deformation strain of 0.8 were used. Stress wave separation technology was used for data analysis [20]; the engineering stress-engineering strain curves were also obtained for comparison with the LSRCT results.

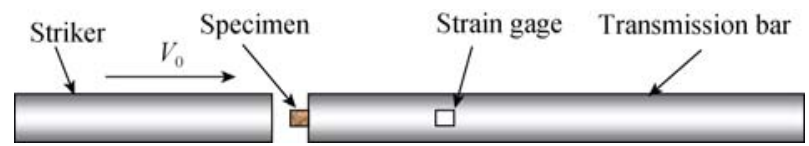

Fig. 5. Schematic diagram of the direct-impact split Hopkinson pressure bar ( $V_{0}$ is the original impact speed of striker).

To analyze the evolution of the pore structure and to investigate the deformation mechanism, the segmented strain method was used; i.e., the specimens were compressed to strains of $0.05,0.30,0.45$, and 0.75 and then unloaded. The deformed specimens with various compressive strains were cut along the compressive direction for analysis of their pore structure. Notably, to control the deformation strain in the HSRCTs, spacing rings with different heights were used to limit the stop position of the striker, as shown in Fig. 4(b). In addition, the compressive deformation process during the HSRCTs was also recorded by a high-speed camera for analysis of the dynamic deformation process and the deformation mechanism.

\section{Results and discussion}

\subsection{Compressive stress-strain curves}

Fig. 6 shows a typical compressive stress-strain curve of lotus-type porous copper. Similar to the case of other porous materials, the compressive stress-strain curve can be divided into three stages: the linear elastic stage, the plateau stage, and the densification stage. The linear elastic stage occurs at low strain levels, commonly less than 0.005 . In this stage, the stress elevates linearly with increasing strain. In the plateau stage, the stress increases slowly in a wide strain range from 0.005 to approximately 0.55 . When the strain exceeds 0.55 , the curve shows the densification stage with the characteristics of a rapid increase of stress with increasing strain. The characteristic parameters, including the plateau stress $\sigma_{\mathrm{pl}}$ and the densification initial strain $\varepsilon_{\mathrm{cd}}$, can be evaluated from the stress-strain curve; these parameters are two of the most important mechanical parameters for porous materials. When porous materials suffer a compressive load or impact, the $\sigma_{\mathrm{pl}}$ and $\varepsilon_{\mathrm{cd}}$ determine their cushioning capacity. When they are used as energy-absorbing components, the $\sigma_{\mathrm{pl}}$ and $\varepsilon_{\mathrm{cd}}$ can characterize the energy-absorbing efficiency of the materials. Compared with foam structures, lotus-type porous structures exhibit much larger $\sigma_{\mathrm{pl}}$ values, which indicates better energy-absorbing properties.

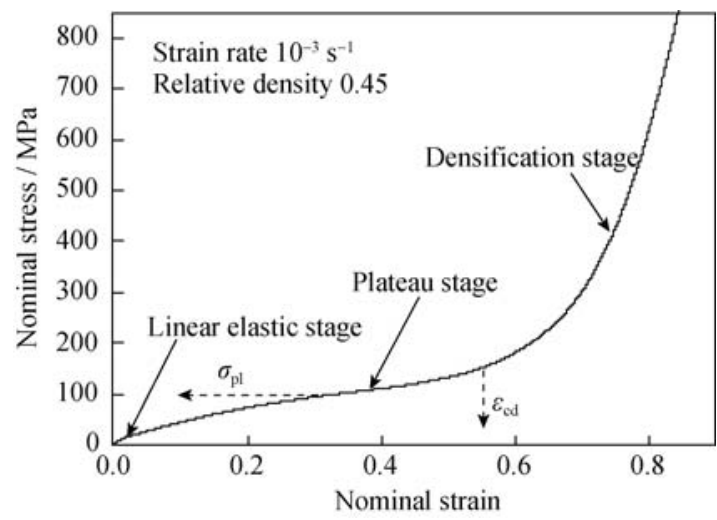

Fig. 6. Typical compressive stress-strain curve of lotus-type porous copper.

\subsection{Effect of strain rate on the compressive stress-strain curves}

The compressive stress-strain curves of the lotus-type porous copper measured at various strain rates are summarily plotted in Fig. 7. The results in Fig. 7 indicate that the compressive stress-strain curves of the lotus-type porous copper exhibit the following characteristics: First, all of the curves obviously include three stages (i.e., the linear elastic stage, the plateau stage, and the densification stage). Second, with an increasing strain rate, the stress-strain curves elevate in the plateau and densification stages. When the strain rate is $10^{-1}$ or less, the strain rate has little influence on the stress-strain curve. When the strain rate exceeds $10^{-1}$, the influence increased, which means that the strain rate sensi- 
tivity was enhanced with the increase in strain rate. Finally, the strain rate sensitivity differs in the three strain ranges.

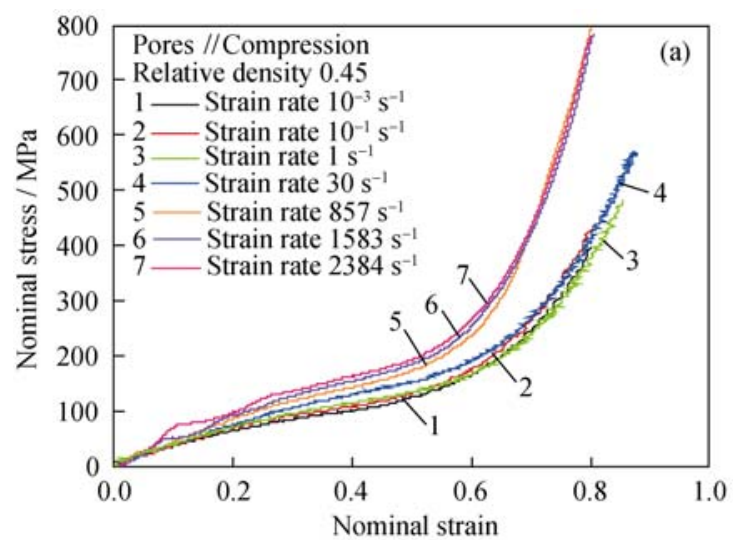
The strain rate shows a strong effect on the stress-strain

curve in the strain range of $0.05-0.7$, which corresponds to the plateau stage, and has little influence in the linear and densification stages.

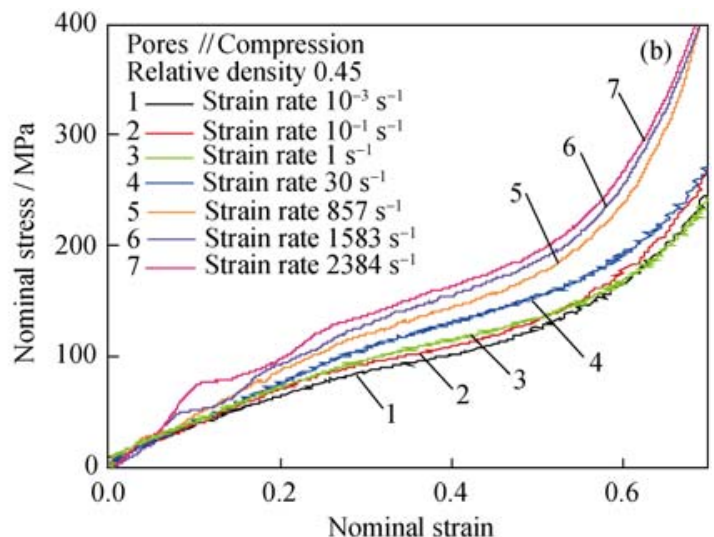

Fig. 7. Stress-strain curves of lotus-type porous copper compressed along the LD at various strain rates; (b) is the enlarged image of (a).

\subsection{Effect of strain rate on the plateau stress and the densification initial strain}

The energetics method was used in this work to calculate the $\varepsilon_{\text {cd }}$. According to Eq. (3), the $\omega-\varepsilon$ curve can be acquired from the compressive stress-strain curve. As shown in Fig. 8 , the strain corresponding to the highest $\omega$ value is defined as the densification initial strain. Here, the value of $\omega$ reflects the energy-absorbing efficiency of porous materials. When densification of porous materials starts, the energy-absorbing efficiency begins to decrease; thus, the strain corresponding to the highest value of $\omega\left(\omega_{\max }\right)$ is just the $\varepsilon_{\mathrm{cd}}$.

$\omega(\varepsilon)=\frac{1}{\sigma(\varepsilon)} \int_{0}^{\varepsilon} \sigma(\varepsilon) \mathrm{d} \varepsilon$

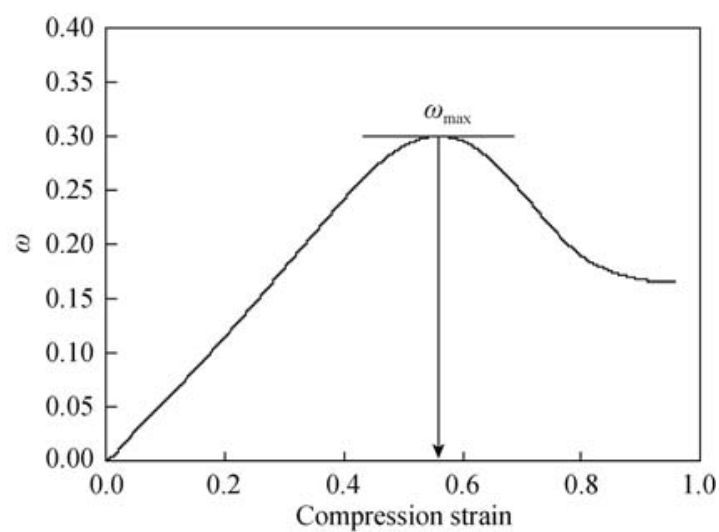

Fig. 8. Schematic diagram of the energetic method used to calculate the densification initial strain of the lotus-type porous copper.

By calculating the densification initial strain, we observed that the $\varepsilon_{\mathrm{cd}}$ values of the lotus-type porous copper all fell within the range of 0.5 to 0.6 at a low or high strain rate. Meanwhile, regarding the characteristics of the stress-strain curves in Fig. 6, the plateau stress would be influenced by the higher curve slope when the strain was less than 0.1 , therefore, the average stress in the strain range of $0.1-0.6$ was regarded as the plateau stress and it was calculated by Eq. (4):

$\sigma_{\mathrm{pl}}=\frac{\int_{\varepsilon_{0.1}}^{\varepsilon_{0.6}} \sigma(\varepsilon) \mathrm{d} \varepsilon}{\varepsilon_{0.6}-\varepsilon_{0.1}}$

To compare the lotus-type porous copper with foam metals, a general analysis method for foam metals was employed in the present work [11], i.e., the degrees of relative change in $\sigma_{\mathrm{pl}}$ and $\varepsilon_{\mathrm{cd}}$ at a certain strain rate were compared with those corresponding to a static deformation condition. If the change is greater than $20 \%$, the porous copper deformed at this strain rate can be treated as exhibiting strain-rate sensitivity. In the present work, the deformation at a strain rate of $10^{-3} \mathrm{~s}^{-1}$ was approximately treated as the static deformation condition and the plateau stresses and densification initial strains of the lotus-type porous copper at various strain rates are listed in Table 1. The results in Table 1 imply that the plateau stress of the lotus-type porous copper with a relative density of 0.45 along the LD exhibits strain-rate sensitivity at a strain rate of $30 \mathrm{~s}^{-1}$ or greater. The strain rate also affects the densification initial strain of the lotus-type porous copper, i.e., the densification initial strain at a high strain rate is less than that at a low strain rate, which indicates that densification stage starts earlier at high strain rates. 
Table 1. Plateau stresses and densification initial strains of the lotus porous copper at various strain rates

\begin{tabular}{ccccc}
\hline \multirow{2}{*}{$\begin{array}{c}\text { Strain } \\
\text { rate } / \mathrm{s}^{-1}\end{array}$} & $\begin{array}{r}\sigma_{\mathrm{pl}} / \\
\mathrm{MPa}\end{array}$ & $\frac{\sigma_{\mathrm{pl}}-\sigma_{\mathrm{pl}}^{10^{-3}}}{\sigma_{\mathrm{pl}}^{10^{-3}}} \times 100$ & $\varepsilon_{\mathrm{cd}}$ & $\frac{\varepsilon_{\mathrm{cd}}-\varepsilon_{\mathrm{cd}}^{10^{-3}}}{\varepsilon_{\mathrm{cd}}^{10^{-3}}} \times 100$ \\
\hline $10^{-3}$ & 83.00 & 0.00 & 0.60 & 0.00 \\
$10^{-1}$ & 89.79 & 8.18 & 0.61 & 1.67 \\
1 & 94.08 & 13.35 & 0.60 & 0.00 \\
30 & 106.95 & 28.86 & 0.63 & 5.00 \\
857 & 117.03 & 41.00 & 0.54 & -10.00 \\
1583 & 124.32 & 49.78 & 0.55 & -8.33 \\
2384 & 133.91 & 61.34 & 0.56 & -6.67 \\
\hline
\end{tabular}

\subsection{Compressive deformation mechanism}

Some mechanisms have been proposed to explain why porous metals exhibit strain-rate sensitivity during compression deformation. One such mechanism indicates that the increase of plateau stress at high strain rates might be related to the gas in the pores of porous materials. When porous materials are compressed at low strain rates, the gas in the pores has sufficient time to escape. Therefore, the strain rate has little influence on the plateau stress. However, when the strain rate is sufficiently high, the gas has no time to escape, thus an instantaneous increase of atmospheric pressure will result in an enhancement of the plateau stress. Contrarily, Deshpande and Fleck [11] have hypothesized that the gas has little influence on the strain-rate sensitivity on the basis of their investigation of the compressive behaviors of foam metals with open or closed pores. Given that lotus-type porous copper has greater accessible porosity, the escape of gas during compressive deformation is advantageous. Therefore, the gas in the pores would have little influence on the strain-rate sensitivity of the lotus-type porous copper.

Another proposed mechanism indicates that the plateau stress of porous materials can be controlled by the deformation or failure mechanism of the pore walls, including elastic buckling, plastic buckling, or brittle fracture [2]. The change of plateau stress with variation of the strain rate is attributed to the influence of strain rate on the failure mechanism of the pore wall, and the shockwave effect, in particular, may be responsible for the difference in deformation modes under dynamic deformation conditions. During deformation under high-speed impact, the shockwave spreading effect causes the deformation of porous materials behind the wave. Thus, local deformation bands are generated near the end face of the specimen by the impaction of the pressure bar and then spread along the LD of the specimen, resulting in an inhomogeneous deformation in porous materials. To ob- serve the shockwave effect, we used high-speed photography to record the deformation process of lotus-type porous copper at high strain rates, as shown in Fig. 9.

The images in Fig. 9 reveal that no local deformation band was formed on the specimen during the impact deformation process. The compressive deformation behavior at high strain rate is similar to that at low strain rate. The upsetting ring is homogenous and becomes obviously drum-shaped during the deformation process. Therefore, the shockwave effect is not the main cause of the strain-rate sensitivity of the lotus-type porous copper at high strain rates.

To investigate the mechanism by which the strain rate influences the mechanical behavior of lotus-type porous copper, we used the strain controlling method to investigate in detail the deformation process and the evolution of pore structures at both low and high strain rates along the LD; the results are presented in Fig. 10 and Fig. 11, respectively.

The images in Fig. 10 indicate that, during the LSRCD for the pores with inhomogeneous wall thickness, local bending deformation previously occurs at some thin positions of the walls. However, in the case of pores with homogenous wall thickness, plastic deformation spreads by periodic S-shaped bending, as shown in Fig. 10(e). Bent pore walls fold onto each other and fill into adjacent pores to induce densification of the porous materials. Because of the low deformation rate, the S-shaped bending deformation process has sufficient time to complete. During the S-shaped bending deformation, the pore walls mainly deform in adjacent pores and then contact each other at a high deformation strain. Before the pore walls contact each other, deformation resistance mainly derives from plastic bending (instead of flow resistance caused by upsetting the deformation of pore walls), which results in the deformation resistance slowly increasing. As the densification stage is approached, the flow stress induced by plastic deformation of dense metals causes a rapid increase in the deformation resistance; this process is consistent with the stress-strain curves in Fig. 7.

When the strain rate is high, local bending deformation also occurs at the thin positions of the walls of pores with inhomogeneous pore-wall thinness. However, this behavior at high strain rates differs from that at low strain rates in that a C-shaped bending deformation occurs instead of S-shaped bending deformation because the deformation time is too short to accommodate S-shaped bending, as shown in Fig. 11(e). Because $\mathrm{C}$-shaped walls have larger radii than S-shaped walls, the bent pore walls contact other walls at a smaller deformation strain, resulting in strain hardening at elevated strain rates. Thus, as shown in Fig. 7, the $\sigma_{\mathrm{pl}}$ is 

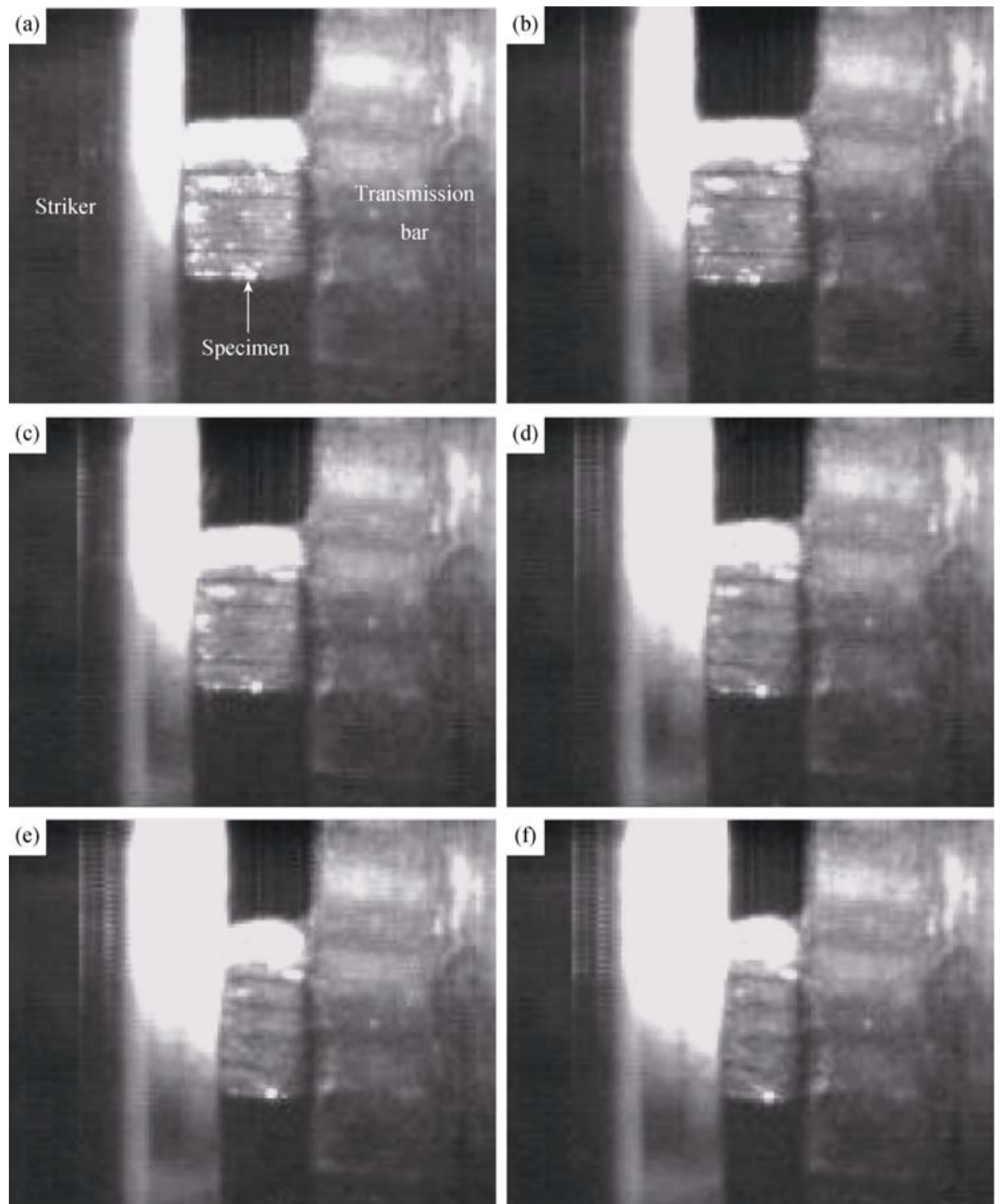

Fig. 9. Compressive deformation process of lotus-type porous copper at a strain rate of $1583 \mathrm{~s}^{-1}$ recorded by a high-speed camera with a shooting speed of 50000 frames per second.
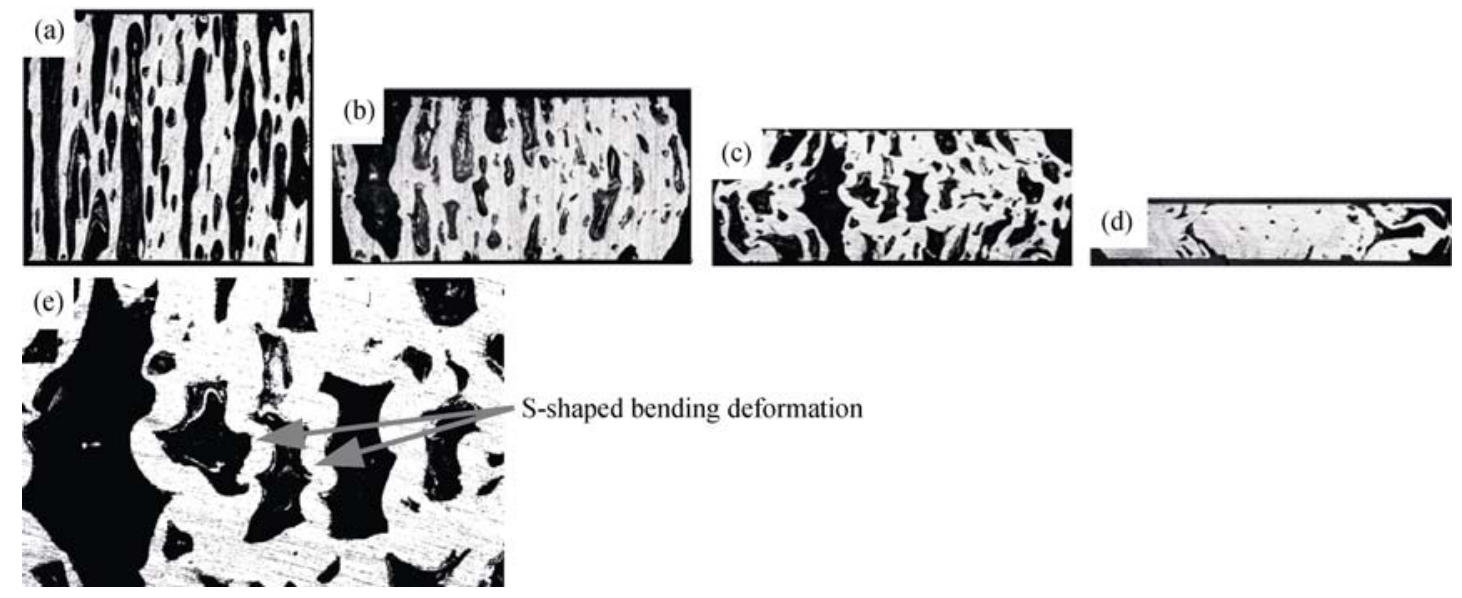

Fig. 10. Longitudinal-sectional morphologies of lotus-type porous copper deformed at strains of (a) 0.05 , (b) 0.30 , (c) 0.45 , and (d) 0.75 with a strain rate of $10^{-3} \mathrm{~s}^{-1}$ along the $\mathrm{LD}$; (e) is the enlarged image of (c). 


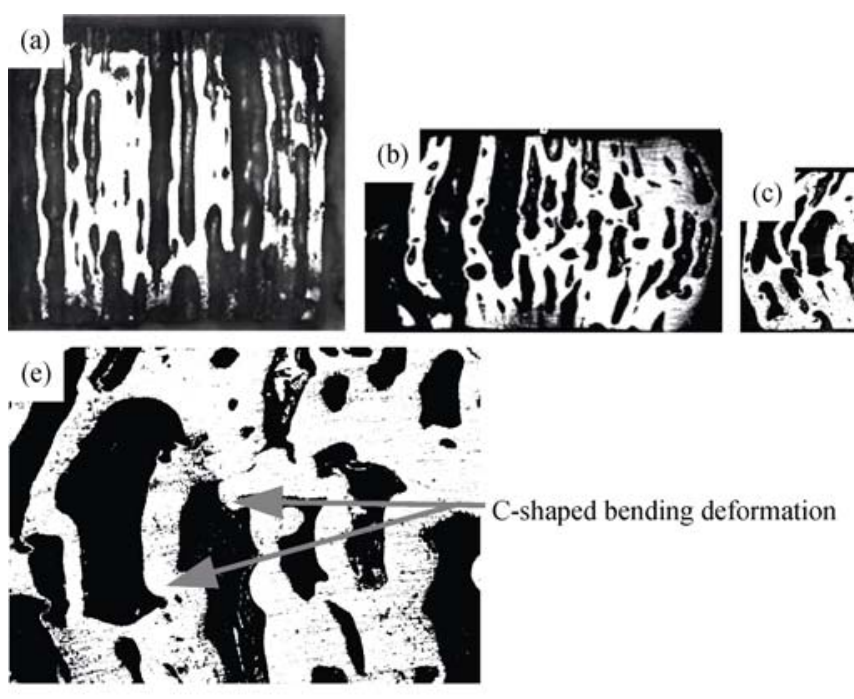

Fig. 11. Longitudinal-sectional morphologies of lotus-type porous copper deformed at a strain of (a) 0.05 , (b) 0.30 , (c) 0.45 , and (d) 0.75 with a strain rate of $1583 \mathrm{~s}^{-1}$ along the $L D$; (e) is the enlarged image of (c).

strongly enhanced with the increase in strain rate, i.e., strain-rate sensitivity is observed when the strain rate exceeds a certain value because the deformation modes change from $\mathrm{S}$-shaped bending to $\mathrm{C}$-shaped bending. The critical strain rate for strain-rate sensitivity is simply the strain rate at which the deformation mode changes. Fig. 12 summarizes the three types of deformation modes. During LSRCD, local banding (Fig. 12(c)) and S-shaped bending (Fig. 12(b)) occur, whereas C-shaped bending (Fig. 12(a)) and local banding occur during HSRCD.

The densification initial strain is closely related to the deformation degree of pore walls. Before the densification stage, sufficient space exists among pore walls and the walls can bend unrestrictedly. As the degree of deformation increases, bent walls fill into the pores gradually. When the compress deformation strain reaches $\varepsilon_{\mathrm{cd}}$, the bending deformation of the pore walls is basically complete; the bent pore walls fold and contact each other, which causes a rapid increase in stress and indicates the beginning of the densification stage. The results in Table 1 indicate that, when compressed at a low strain rate, the densification initial strain varied within a narrow range, but the densification initial strain was larger than when the specimen was compressed at high strain rates. This behavior results from the different deformation behaviors at various strain rates. As previously mentioned, when the lotus-type porous copper specimens were compressed at low strain rates, the deformation modes of their pore walls exhibited S-shaped deformation. Because of the lower deformation rate of the lotus-type porous copper, the deformed pore walls could be filled into the clearance sufficiently and led to a greater degree of densification.
However, when compressed at high strain rates, the pore walls of the lotus-type porous copper deformed in C-shaped mode. Because of the greater deformation rate, the filling process of the deformed pore walls into the clearance was not sufficient, and the degree of compressed densification was diminished.

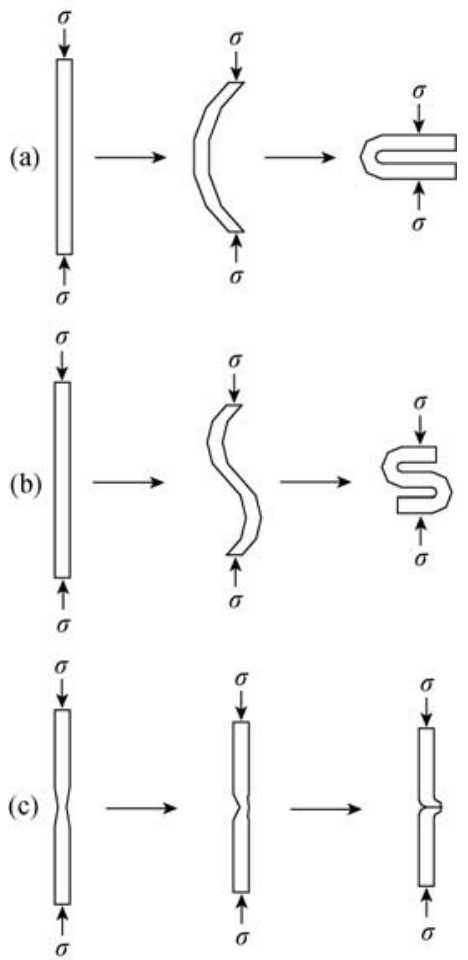

Fig. 12. Schematic diagrams of three deformation modes of pore walls in lotus-type porous copper during compressive deformation along the LD: (a) C-shaped bending; (b) S-shaped bending; (c) local bending. 


\section{Conclusions}

(1) All of the compressive stress-strain curves of lotus-type porous copper acquired at various strain rates consisted of three stages: the linear elastic stage, the plateau stage, and the densification stage.

(2) At low strain rates $\left(<1.0 \mathrm{~s}^{-1}\right)$, the strain rate had little influence on the stress-strain curves. However, when the strain rate exceeded $1.0 \mathrm{~s}^{-1}$, a significant influence of the strain rate on the plateau stage was observed and exhibited obvious strain-rate-hardening characteristics; in addition, the strain rate also influenced the densification initial strain.

(3) When the strain rate exceeded $30 \mathrm{~s}^{-1}$, the change of the deformation mode at low and high strain rates was the main mechanism for the strain-rate sensitivity of the plateau stress on the compressive stress-strain curves of lotus-type porous copper, whereas the shockwave effect had little influence.

\section{Acknowledgements}

The present work was financially supported by the National Natural Science Foundation (No. 50904004).

\section{References}

[1] J. Banhart, Manufacture, characterisation and application of cellular metals and metal foams, Prog. Mater. Sci., 46(2001), No. 6, p. 559.

[2] L.J. Gibson and M.F. Ashby, Cellular Solids: Structure and Properties, 2nd Ed., Cambridge University Press, Cambridge, 1999, p. 6.

[3] H.P. Degischer, B. Kriszt. X.Q. Zuo, and Y. Zhou, Handbook of Cellular Metals, Chemical Industry Press, Beijing, 2005, p. 221.

[4] V.I. Shapovalov, Method for Manufacturing Porous Articles, U. S. Patent, Appl. 5181549, 1993.

[5] H. Nakajima and T. Ide, Fabrication of porous copper with directional pores through thermal decomposition of compounds, Metall. Mater. Trans. A, 39(2008), No. 2, p. 390.

[6] H. Nakajima, Fabrication, properties and application of porous metals with directional pores, Prog. Mater. Sci., 52(2007), No. 7, p. 1091.

[7] H. Nakajima, T. Ikeda, and S.K. Hyun, Fabrication of lo- tus-type porous metals and their physical properties, $A d v$. Eng. Mater., 6(2006), No. 6, p. 377.

[8] S.K. Hyun and H. Nakajima, Anisotropic compressive properties of porous copper produced by unidirectional solidification, Mater. Sci. Eng. A, 340(2003), No. 1-2, p. 258.

[9] T. Ichitsubo, M. Tane, H. Ogi, M. Hirao, T. Ikeda, and H. Nakajima, Anisotropic elastic constants of lotus-type porous copper: measurements and micromechanics modeling, Acta Mater., 50(2002), No. 16, p. 4105.

[10] T. Mukai, H. Kanahashi, K. Higashi, T. Miyoshi, M. Mabuchi, and T.G. Nieh, Experimental study of energy absorption in a close-celled aluminum foam under dynamic loading, Scripta Mater., 40(1999), No. 8, p. 921.

[11] V.S. Deshpande and N.A. Fleck, High strain rate compressive behaviour of aluminium alloy foams, Int. J. Impact Eng., 24(2000), No. 3, p. 277.

[12] K.A. Dannemann and J.J. Lankford Jr., High strain rate compression of closed-cell aluminium foams, Mater. Sci. Eng. A, 293(2000), No. 1-2, p. 157.

[13] C.K.H. Dhararn and F.E. Hauser, Determination of stressstrain characteristics at very high strain rates, Exp. Mech., 10(1970), No. 6, p. 370.

[14] D.P. Mondal, M.D. Goel, and S. Das, Compressive deformation and energy absorption characteristics of closed cell aluminum-fly ash particle composite foam, Mater. Sci. Eng. A, 507(2009), No. 1-2, p. 102.

[15] K. Koichi and T. Yoshihiro, Strain rate sensitivity and energy absorption of Zn-22Al foams, Scripta Mater., 55(2006), No. 6, p. 501.

[16] Y. Feng, Z.G. Zhu, F.Q. Zu, S.S. Hu, and Y. Pan, Strain rate effects on the compressive property and the energy-absorbing capacity of aluminum alloy foams, Mater. Charact., 47(2001), No. 5, p. 417.

[17] D. Yao, X.H. Liu, X.F. Liu, and J.X. Xie, Axial compressive deformation behaviors and constructive relation for lotus-type porous copper, Chin. J. Nonferrous Met., 18(2008), No. 11, p. 1995.

[18] X.H. Liu, D. Yao, X.F. Liu, and J.X. Xie, Deformation behaviors and constructive relation of lotus-type porous copper under compressive direction perpendicular to pores, Chin. J. Nonferrous Met., 19(2009), No. 7, p. 1237.

[19] D.A. Gorham, P.H. Pope, and J.E. Field, An improved method for compressive stress-strain measurements at very high strain rates, Proc. R. Soc. Lond. A, 438(1992), No. 1902, p. 153.

[20] S.W. Park and M. Zhou, Separation of elastic waves in split Hopkinson bars using one-point strain measurements, Exp. Mech., 39(1999), No. 4, p. 287. 